\title{
Solving a weighty problem: systematic review and meta-analysis of nutrition interventions in severe mental illness ${ }^{\dagger}$
}

\author{
Scott B. Teasdale, Philip B. Ward, Simon Rosenbaum, Katherine Samaras and Brendon Stubbs
}

\section{Background}

Nutrition interventions would appear fundamental for weight management and cardiometabolic risk reduction in people experiencing severe mental illness (SMI). comprehensive evaluation of nutrition interventions is lacking.

\section{Aims}

To subject randomised controlled trials of nutrition interventions in people with SMI to systematic review and meta-analysis, and to measure anthropometric and biochemical parameters and nutritional intake.

\section{Method}

An electronic database search identified trials with nutrition intervention components. Trials were pooled for meta-analysis. Meta-regression analyses were performed on anthropometric moderators.

\section{Results}

Interventions led to significant weight loss (19 studies), reduced body mass index (17 studies), decreased waist circumference (10 studies) and lower blood glucose levels (5 studies). Dietitian-led interventions (6 studies) and studies delivered at antipsychotic initiation (4 studies) had larger effect sizes.

\section{Conclusions}

Evidence supports nutrition interventions as standard care in preventing and treating weight gain among people experiencing SMI.

\section{Declaration of interest}

None.

\section{Copyright and usage}

(c) The Royal College of Psychiatrists 2017.
People with severe mental illness (SMI) consume diets that are more energy-dense, highly processed, higher in salt and contain less fruit and vegetables, compared with the general population. ${ }^{1}$ People with SMI also engage in lower levels of physical activity, ${ }^{2}$ and have higher rates of smoking and substance use. ${ }^{3,4}$ Antipsychotic medications induce greatly increased hunger, decreased satiety and increased cravings for sweet foods and drinks., Additionally, a number of adverse eating styles have been observed, including fast-eating syndrome, disordered eating habits (e.g. only eating one main meal daily), increased consumption of junk food and low food literacy. ${ }^{5-7}$ Although the poor physical health of people with SMI is well established, consensus on the appropriate prevention and/or treatment interventions is in evolution, with calls for increased emphasis on lifestyle interventions aimed at reducing overweight/obesity and consequent metabolic abnormalities in established $\mathrm{SMI},{ }^{8}$ and preventing these adverse health trajectories in the early stages of psychosis. ${ }^{9,10}$ The mandate for improved physical healthcare and physical health protection in severe mental illness has led to an international working group and declaration, Healthy Active Lives (HeAL), that has established goals for the prevention of cardiometabolic decline in first-episode psychosis (www.iphys.org.au). ${ }^{11}$ Strong evidence now exists for holistic lifestyle interventions, ${ }^{8}$ and as part of this the inclusion of physical activity interventions for people living with severe mental illness. ${ }^{12}$

Poor physical health in people experiencing SMI stems from both modifiable and non-modifiable risk factors linked to the illness itself, compounded by significant medication effects. Antipsychotic medications induce rapid weight gain with associated metabolic abnormalities. ${ }^{13,14}$ This weight gain contributes to the high rates of overweight and obesity and metabolic complications in people with established SMI, with diabetes and hypercholesterolaemia rates respectively two and five

'See editorial, pp. 94-95, this issue. times higher than those observed in the general population. ${ }^{15-18}$ To date, the efficacy of specific components, including modes of delivery, of nutrition interventions has not been systematically evaluated. With irrefutable evidence demonstrating the crucial role of nutrition in weight management, ${ }^{19}$ and the prevention and treatment of metabolic disease in the general population, ${ }^{20,21}$ a comprehensive assessment of various nutrition intervention strategies employed to assist a highly vulnerable and challenging populations is a priority. The specific questions to be answered by this review and meta-analysis of randomised controlled trials (RCTs) were the following.

(a) Do nutrition interventions improve anthropometric measures (weight, body mass index and waist circumference) and biochemical profiles (lipids, glucose and insulin) of people living with SMI?

(b) Do nutrition interventions improve the nutritional intake of people living with SMI?

\section{Method}

The aims and method of this systematic review and meta-analysis were registered with the PROSPERO database prior to conducting the review (CRD42014014017). Reporting was conducted in accordance with the Preferred Reporting Items for Systematic Reviews and Meta-Analyses (PRISMA) statement. ${ }^{22}$

\section{Search strategy}

An electronic database search was completed from earliest record to February 2015 using Medline, EMBASE, the Cochrane Central Register of Clinical Trials, PsycINFO and CINAHL, using key nutritional, anthropometric and psychiatric terminology. Google Scholar and relevant published systematic reviews were manually searched for additional titles. Study eligibility was assessed according to inclusion criteria by two investigators. If agreement 
was not established, a third investigator acted as arbitrator. Data were extracted by the two investigators and pooled for meta-analysis.

\section{Quality assessment}

Trial quality was assessed using a modified version of the Critical Appraisal Skills Programme (CASP) Randomised Controlled Trial Checklist. ${ }^{23}$ Trial characteristics were assessed across four criteria: concealed allocation, assessor masking, treatment equality between groups (excluding intervention) and accounting for all participants randomised. One point was allocated for each criterion, giving a maximum score of four.

\section{Participants}

Randomised controlled trials recruiting participants 18 years old or over meeting DSM or ICD criteria for SMI (schizophrenia spectrum disorder, bipolar affective disorder, depression with psychotic features) were eligible for inclusion. There was no restriction on medication use.

\section{Interventions}

Studies of stand-alone nutrition interventions or nutrition interventions delivered as part of a multidisciplinary intervention were included. Interventions comprising individualised nutrition counselling, group nutrition education, shopping or cooking classes were eligible. No restriction was placed on intervention setting: interventions in in-patient services, out-patient programmes and community volunteer services or otherwise were included. The process of referral to the study, location where the intervention was delivered, and the professional background of those who delivered the intervention were recorded. No restriction was placed on intervention intensity or duration.

\section{Outcome measures}

All trials that met inclusion criteria were included in a qualitative analysis. Trials were included in the meta-analysis if they provided adequate data on anthropometric (primary outcomes: weight, body mass index and waist circumference) and biochemical and/or nutritional parameters (secondary outcomes: total cholesterol, low-density lipoprotein, high-density lipoprotein, triglycerides, blood glucose, insulin and dietary intake). Data collection time points included pre- and post-intervention and follow-up. Where necessary, corresponding authors of included trials were contacted to provide additional data for inclusion in the meta-analysis. A follow-up and final email was sent 3 weeks later if corresponding authors did not reply to the initial request.

\section{Statistical analysis}

Because of the anticipated heterogeneity we used a random effects meta-analysis and calculated Hedges' $g$ and 95\% confidence intervals as the effect size measure. The meta-analysis was conducted in the following stages. First, we calculated Hedges' $g$ and the $95 \%$ CI for the primary outcome measures: weight, body mass index (BMI) and waist circumference. Second, we conducted subgroup analyses to investigate differences in the primary outcomes for the main analysis according to whether interventions were delivered by a dietitian or not, and whether they were delivered at initiation of antipsychotic therapy $(\leqslant 3$ months exposure to second-generation antipsychotic medication) or subsequent to antipsychotic use. For each subgroup analysis we investigated the between-subgroup difference in effect size and report the corresponding $P$ value. Third, we conducted metaregression analyses investigating potential moderators of the primary outcome results including the percentage of men and mean age in both control and intervention groups, percentage receiving antipsychotics, duration of intervention, the profession delivering the intervention and exercise intensity. In order to test whether the profession of the person who delivered the intervention (dietitian $v$. other healthcare professional) was an independent predictor of our primary outcomes from other variables (in particular, exercise participation in multimodal programmes), we conducted multivariate meta-regressions. In order to correct for multiple testing of covariates in our metaregression, a Bonferroni correction was made and a new $P$ value to indicate significance was set at 0.006 (0.05 divided by 8 ). In the next stage, we calculated Hedges' $g$ and $95 \%$ CI for the secondary outcomes including systolic and diastolic blood pressure, total cholesterol, high-density lipoprotein (HDL), lowdensity lipoprotein (LDL), triglycerides, blood glucose and insulin. We investigated heterogeneity with the $I^{2}$ statistic. Publication bias was assessed with a visual inspection of funnel plots and with the Begg-Mazumdar Kendall's tau and Egger bias test. $^{24,25}$ If we encountered publication bias, we adjusted for this by conducting a trim and fill adjusted analysis to remove the most extreme small studies from the positive side of the funnel plot, ${ }^{26}$ and recalculated the effect size at each iteration until the funnel plot was symmetrical about the (new) effect size. All analyses were conducted with Comprehensive Meta-Analysis software version 3.

\section{Results}

After accounting for duplicates, 7045 unique records were screened from the database searches. Full-text articles were accessed for 176 records; 150 did not meet the inclusion criteria and were subsequently excluded (Fig. 1). Twenty-six studies met the inclusion criteria, ${ }^{27-52}$ but 6 studies reported incomplete data and could not be pooled for meta-analysis. ${ }^{31,32,36,41,46,48}$ For the primary outcomes measures, 19 studies were pooled for weight, $27-30,33-35,37,38,40,42-45,47,49-5217$ studies for $\mathrm{BMI},{ }^{27-29,33-35,37,39,40,42-45,47,50-52}$ and 11 studies for waist circumference. ${ }^{28,33-35,37,39,40,44,47,50,52}$ For secondary outcomes 8 studies were pooled reporting the impact of nutrition interventions on biochemical outcomes (blood pressure, lipids, glucose, insulin). ${ }^{28,29,34,37,39,42,45,47}$ Measures of nutritional intake were reported in 6 studies. $^{28,31,32,38,44,46}$ These measures could not be pooled for quantitative analysis, but were included in qualitative analysis. Longer-term follow-up of 2 included studies was reported in separate publications. ${ }^{53,54}$ Comprehensive datasets were obtained directly from corresponding authors for 2 studies. $^{37,42}$

\section{Characteristics of included trials}

The sample size within studies ranged from $n=15$ to $n=291$. Mean participant age ranged from 26 years $($ s.d. $=15.5)$ to 54.8 years (s.d.=8.2). Diagnoses within included studies were schizophrenia (18 studies), schizoaffective disorder (12 studies), schizophreniform disorder (3 studies), bipolar affective disorder (7 studies), delusional disorder (2 studies), brief reactive psychosis (2 studies), psychosis not otherwise specified (2 studies), personality disorder (2 studies), anxiety (2 studies) and depression (2 studies). First-episode psychosis, major affective illness, major depressive disorder, psychotic depression and post-traumatic stress disorder were identified in one study each. More general diagnostic descriptors were also employed in a minority of studies: SMI (4 studies), psychosis (2 studies), schizophrenia spectrum (1 study) and autism spectrum (1 study). Participants were recruited from out-patient settings (21 studies), in-patient 


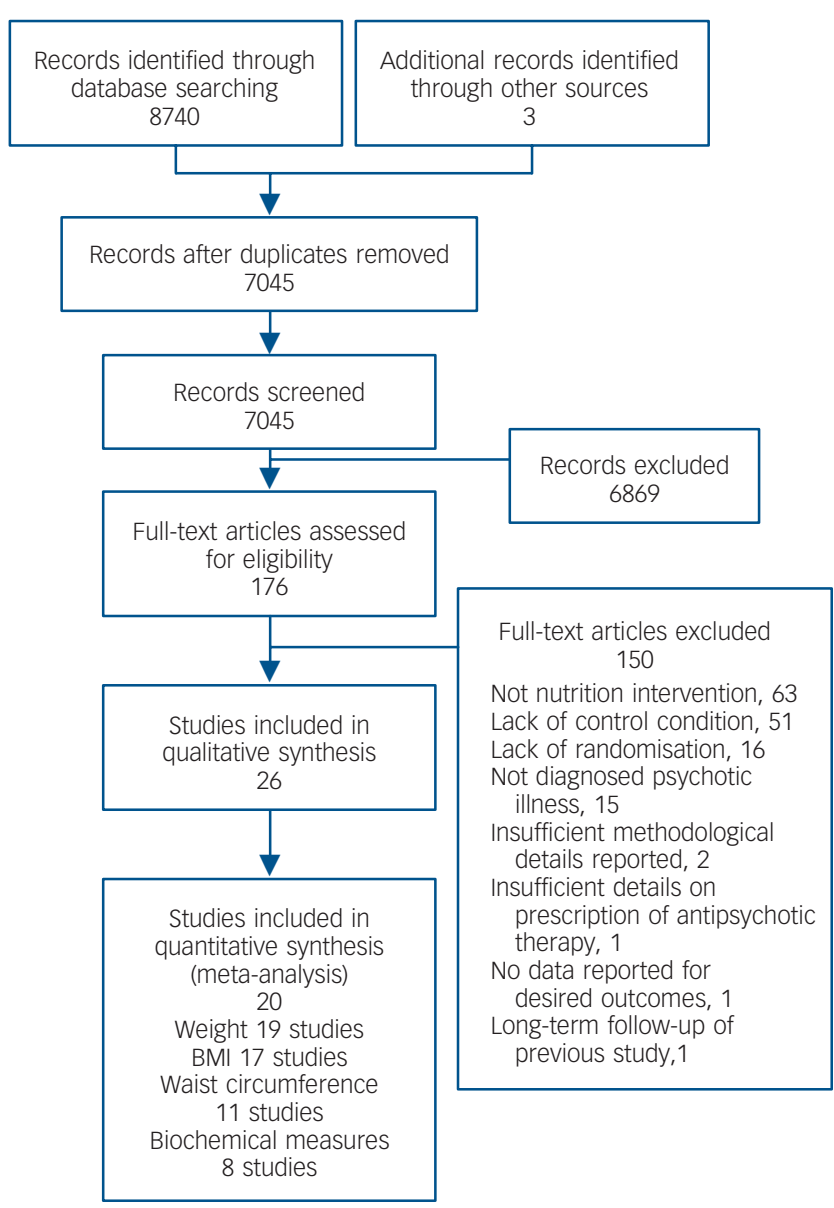

Fig. 1 Flowchart of study search. BMI, body mass index.

settings ( 3 studies) or included a mix of out-patients and in-patients (2 studies). Characteristics of included trials are summarised in online Table DS1.

\section{Interventions}

Nutrition intervention delivery methods included individualised counselling (12 studies), group education (12 studies) and a mixture of group and individual components (2 studies). Seven studies primarily used dietitians to deliver interventions, ${ }^{33,35,42,45,48,49,52}$ with an additional 5 studies including a nutrition professional as a smaller part of the intervention primarily delivered by other clinicians. ${ }^{28,30,37,41,47}$ Fourteen studies did not report input from a nutrition professional. ${ }^{27,29,31,32,34,36,38-40,43,44,46,50,51}$ Seven studies adopted a manual-based lifestyle intervention, ${ }^{27-29,31,32,43,50}$ predominantly delivered by mental health clinicians. Studies delivered predominantly by dietitians involved individualised assessment and intervention approaches. Other interventions included general nutrition education aimed at improving food literacy (not individualised counselling), weight management guidance and healthy-eating education. Cooking classes were reported in 4 studies. ${ }^{33,36,37,41}$ One study incorporated two meal replacements per day, ${ }^{30}$ and another solely assessed the impact of providing free fruit and vegetables to participant households. ${ }^{46}$

All studies described strategies to alter participant behaviour, such as cue elimination, food diary/record-keeping and food sampling, although the specific behaviour change models used were more difficult to identify and interpret. Psychoeducation was described in 7 studies. $^{27,28,33,43-45,49}$ Cognitive-behavioural therapy was identified in 3 studies, ${ }^{29,42,51}$ and social cognitive therapy in 1 study. ${ }^{34}$ Within the cognitive framework, motivational interviewing and more generally motivational counselling/support were commonly used. ${ }^{27,28,31,32,36,37,39,44,50}$ In addition, the 'stages of change' model was described in 2 studies where motivational interviewing was used. ${ }^{39,50}$ Finally, 1 study described the use of behaviour self-management therapy. ${ }^{34}$ Psychoeducation was combined with motivational counselling/ support in 3 studies. ${ }^{27,28,44}$ Control groups received treatment as usual with or without written physical health information.

\section{Outcome measures}

Twenty-five studies assessed anthropometric measures, predominantly weight and BMI. ${ }^{27-45,47-52}$ Additional measures included waist circumference, waist-to-hip ratio and body fat percentage. Eleven studies (42\%) included biochemical outcome measures, predominantly lipids (cholesterol studies and triglycerides), glucose and insulin. ${ }^{28,33,34,37,39,41,42,45,47,51,52}$ Studies including measures of nutritional intake were limited to 6 studies (25\%), which recorded energy intake (kilojoules/calories), servings of food groups (such as fruit and vegetables), macronutrients (including fat and subgroups unsaturated and saturated fat), fibre and portion size. ${ }^{28,31,32,38,44,46}$ In addition, one study used a 'ten good food score.'

\section{Trial quality}

Seven studies (27\%) scored the maximum four points, ${ }^{27,28,31,32,34,40,44} 4$ studies (15\%) scored three, ${ }^{33,36,50,51} 13$ studies (50\%) scored two, ${ }^{29,30,37-39,41-43,45-48,52}$ and 2 studies $(8 \%)$ scored one (see online Table DS2). ${ }^{35,49}$ 'Group treatment equality' and 'all participants being accounted for' were reported in 25 studies (96\%) and 22 studies (85\%) respectively. Methodological uncertainties included 'concealed group allocation' and 'assessor blinding', described in 12 studies (46\%) and 9 studies (35\%) respectively.

\section{Meta-analysis results}

All the meta-analyses results including subgroup analyses are presented in Table 1.

\section{Primary outcomes}

Pooled analysis showed that nutrition interventions were significantly more effective in reducing weight $v$. control (19 studies: $g=-0.39, \quad 95 \%$ CI -0.56 to $-0.21, \quad P<0.001$, $I^{2}=55 \%$; Fig. 2). There was evidence of publication bias (Begg $-0.41, P=0.01$; Egger $-1.7, P=0.08)$, whereas the Duval \& Tweedie trim and fill effect size adjusting for publication bias remained similar and statistically significant $(g=-0.33,95 \%$ CI -0.44 to -0.22$)$. Nutrition interventions also reduced BMI compared with control groups (17 studies: $g=-0.39,95 \%$ CI -0.56 to $-0.22, P<0.001, I^{2}=51 \%$ ) (see online Fig. DS2a). The results remained statistically significant when adjusted for publication bias in the trim and fill analysis $(g=-0.34,95 \%$ CI -0.45 to -0.23$)$. Nutrition interventions were also effective in reducing waist circumference $v$. control (11 studies: $g=-0.27$, $95 \%$ CI -0.42 to $-0.12, P<0.001, I^{2}=17 \%$; see online Fig. DS3a). The results remained statistically significant in the trim and fill analysis $(g=-0.25,95 \% \mathrm{CI}-0.38$ to -0.12$)$.

\section{Subgroup analysis: antipsychotic use}

A larger effect size was found for studies delivered at the start of antipsychotic pharmacotherapy (4 studies: $g=-0.61,95 \% \mathrm{CI}$ 


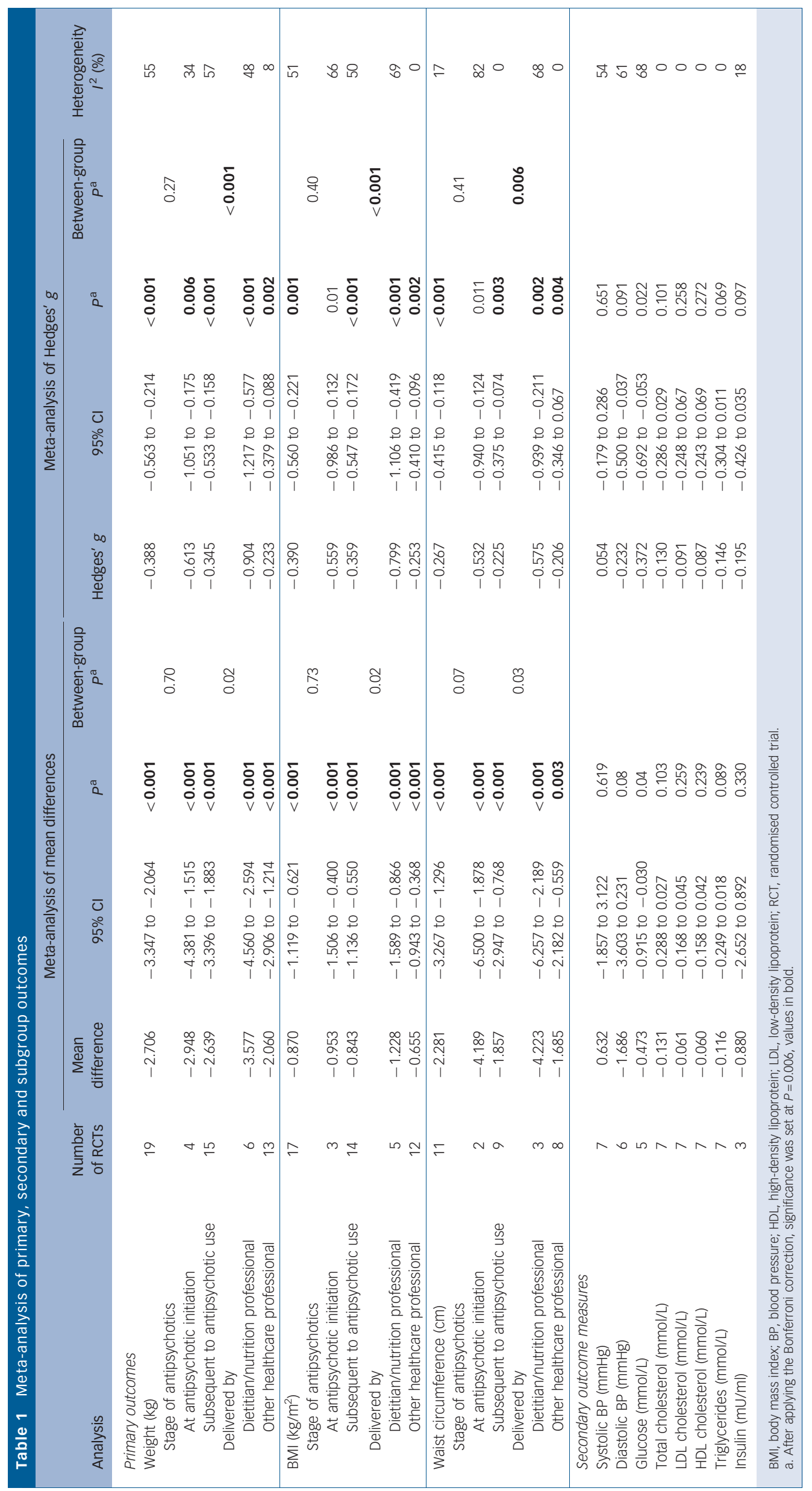


Study

\begin{tabular}{|c|c|c|c|c|}
\hline & \\
\hline & $\begin{array}{l}\text { Hedges' } \\
\quad g\end{array}$ & $\begin{array}{l}\text { Lower } \\
\text { limit }\end{array}$ & $\begin{array}{l}\text { Upper } \\
\text { limit }\end{array}$ & $P$ \\
\hline Goldberg et al (2013) & 0.049 & -0.324 & 0.422 & 0796 \\
\hline Iglesias-Garcia et al (2010) ${ }^{40}$ & 0.000 & -0.981 & 0.981 & 1.000 \\
\hline Cordes et al (2014) & -0.048 & -0.769 & 0.673 & 0.896 \\
\hline Usher et al (2013) ${ }^{50}$ & -0.049 & -0.437 & 0.338 & 0.803 \\
\hline Attux et al $(2013)^{28}$ & -0.078 & -0.425 & 0.270 & 0.662 \\
\hline Lovell et al (2014) ${ }^{44}$ & -0.103 & -0.484 & 0.277 & 0.594 \\
\hline Bron et al $(2011)^{30}$ & -0.107 & -0.520 & 0.306 & 0.612 \\
\hline Gillhoff et al (2010) ${ }^{37}$ & -0.210 & -0.757 & 0.338 & 0.453 \\
\hline Brar et al (2005) ${ }^{29}$ & -0.258 & -0.720 & 0.205 & 0.275 \\
\hline Littrell et al (2003) & -0.268 & -0.733 & 0.198 & 0.259 \\
\hline McKibbin et al (2006) ${ }^{47}$ & -0.440 & -0.959 & 0.078 & 0.096 \\
\hline Daumit et al (2013) $)^{34}$ & -0.463 & -0.715 & -0.211 & 0.000 \\
\hline Scocco et al $(2006)^{49}$ & -0.580 & -1.505 & 0.344 & 0.219 \\
\hline Alvarez-Jimenez et al (2006) ${ }^{27}$ & -0.653 & -1.163 & -0.142 & 0.012 \\
\hline Kwon et al (2006) & -0.770 & -1.434 & -0.107 & 0.023 \\
\hline Weber \& Wyne $(2006)^{51}$ & -0.918 & -1.928 & 0.091 & 0.075 \\
\hline Evans et al (2005) $)^{35}$ & -1.176 & -1.932 & -0.421 & 0.002 \\
\hline Wu et al $(2007)^{52}$ & -1.293 & -1.879 & -0.708 & 0.000 \\
\hline Mauri et al (2008) ${ }^{45}$ & -1.339 & -2.082 & -0.597 & 0.000 \\
\hline & -0.388 & & -0.214 & 0.000 \\
\hline
\end{tabular}

Statistics for each study

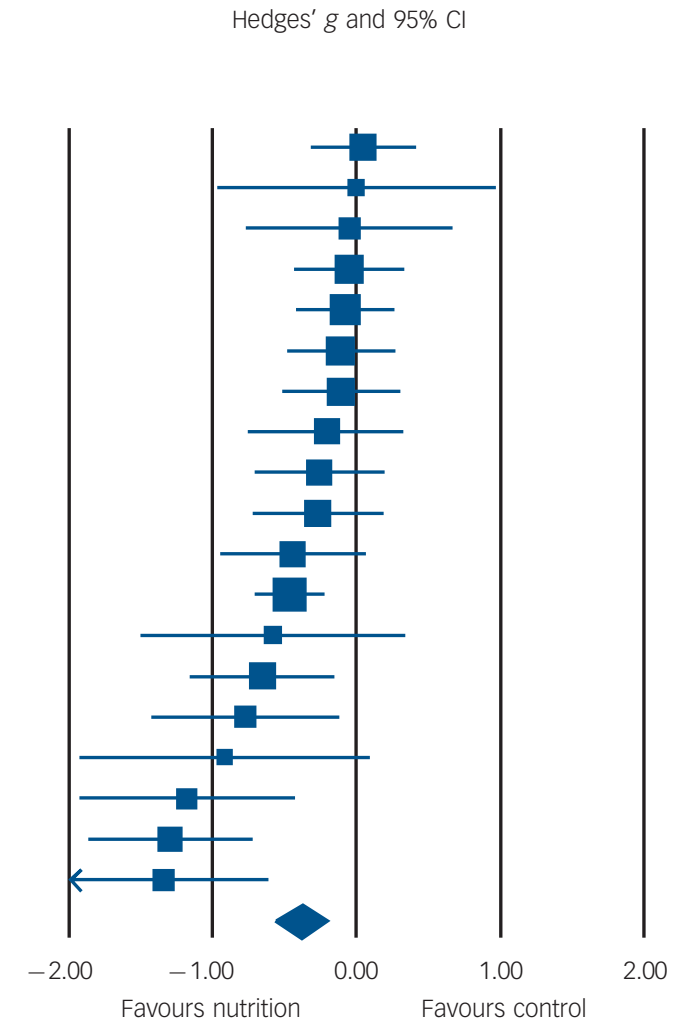

Fig. 2 Effect of nutrition interventions on weight $v$. control group.

-1.02 to $\left.-0.18, P=0.006, I^{2}=34 \%\right)$ compared with studies delivered subsequent to antipsychotic use (15 studies: $g=-0.35$, $95 \%$ CI -0.54 to $-0.16, P<0.001, I^{2}=57 \%$; see online Fig. DS1a). Similar results were seen for BMI $(g=-0.56 v$. $g=-0.36)$ and waist circumference $(g=-0.53$ v. $g=-0.23)$ (see online Figs DS2b and DS3b). Between-group differences did not reach statistical significance after applying a Bonferroni correction (Table 1).

\section{Subgroup analysis: profession delivering intervention}

Subgroup analysis investigating the effect of who delivered the nutrition intervention revealed that dietitians delivering specialised dietary interventions (6 studies: Hedges' $g=-0.90$, $95 \% \mathrm{CI}-1.22$ to $-0.58, P<0.001, I^{2}=48 \%$ ) had a significantly larger effect $(P=0.0005)$ compared with interventions predominantly delivered by other health professionals or mental health clinicians (13 studies: Hedges' $g=-0.23,95 \%$ CI -0.38 to $-0.09, P=0.002, I^{2}=8 \%$ (online Figs DS1b, DS2c and DS3c).

\section{Meta-regression analyses}

weight

Single meta-regression analyses found that the profession delivering the intervention (dietitian $v$. other healthcare professional, $\beta=-0.69,95 \%$ CI -1.05 to $-0.32, P<0.001$ ) was a significant predictor of weight change (Table 2). Multivariate regression analysis found that profession remained a significant predictor of weight change independent of exercise $(\beta=-0.72,95 \%$ CI -1.06 to $-0.37, P<0.001)$. The percentages of men in the control group ( $\beta=0.01,95 \%$ CI 0.0 to $0.02, P=0.05)$ and in the nutrition group ( $\beta=0.02,95 \% \mathrm{CI}-0.0$ to $0.03, P=0.01$ ) were positive predictors of weight (i.e. more difficult to lose weight).

\section{Body mass index}

A dietitian-delivered intervention was a significant moderator of BMI results ( $\beta=-0.53,95 \% \mathrm{CI}-0.9$ to $-0.16, P=0.005)$. This finding was confirmed through multivariate regression analysis and the results remained significant independent of exercise participation $(\beta=-0.52,95 \% \mathrm{CI}-0.85$ to $-0.20, P=0.001)$, with exercise not found to be a significant moderator $(\beta=-0.21,95 \%$ CI -0.44 to $0.02, P=0.07)$.

\section{Waist circumference}

There was a non-significant trend for dietitian-delivered interventions to moderate waist circumference results $(\beta=-0.37$, $95 \%$ CI -0.76 to $-0.02, P=0.06$ ). Multivariate regression analysis confirmed the trend for the profession delivering the intervention as a moderator independent of exercise intensity $(\beta=-0.36,95 \%$ CI -0.76 to $-0.03, P=0.072)$.

\section{Secondary outcomes}

The meta-analyses for the secondary outcomes are presented in Table 1 . The analyses demonstrated that nutrition interventions reduced glucose $(g=-0.37,95 \%$ CI -0.69 to $-0.05, P=0.02$, $I^{2}=68 \%$; online Fig. DS4); however, this was not statistically significant after applying the Bonferroni correction. Triglyceride levels $\left(g=-0.15,95 \% \mathrm{CI}-0.30\right.$ to $\left.0.01, P=0.07, I^{2}=0 \%\right)$ were not significantly affected by nutrition interventions, although trends were evident. Other secondary outcome measures were not significant.

\section{Impact on nutritional intake}

Results for all six studies that assessed changes in nutritional intake favoured the intervention group but could not be pooled for meta-analysis owing to the varying outcomes 


\begin{tabular}{|c|c|c|c|c|}
\hline Moderator & $\beta$ & $95 \% \mathrm{Cl}$ & $P$ & $R^{2}$ \\
\hline \multicolumn{5}{|l|}{ Weight } \\
\hline Mean age control group & 0.007 & -0.012 to 0.027 & 0.456 & 0.06 \\
\hline Mean age nutrition group & 0.002 & -0.018 to 0.021 & 0.874 & 0.00 \\
\hline Percentage men control group & 0.011 & 0.000 to 0.021 & $0.045^{\star}$ & 0.41 \\
\hline Percentage men nutrition group & 0.015 & -0.003 to 0.026 & $0.012^{*}$ & 0.67 \\
\hline Duration of intervention & 0.003 & -0.006 to 0.012 & 0.536 & 0.00 \\
\hline Profession delivering intervention & -0.685 & -1.050 to -0.320 & $<0.001^{* * *}$ & 0.82 \\
\hline \multicolumn{5}{|l|}{ Multivariate analysis } \\
\hline Profession delivering intervention & -0.719 & -1.064 to -0.374 & $<0.001^{* * *}$ & 1.00 \\
\hline Exercise intensity & -0.180 & -0.399 to 0.040 & 0.108 & \\
\hline \multicolumn{5}{|l|}{$B M I$} \\
\hline Mean age control group & 0.002 & -0.017 to 0.021 & 0.826 & 0.00 \\
\hline Mean age nutrition group & -0.002 & -0.020 to 0.017 & 0.868 & 0.09 \\
\hline Percentage men control group & 0.010 & -0.003 to 0.023 & 0.145 & 0.21 \\
\hline Percentage men nutrition group & 0.009 & -0.004 to 0.022 & 0.161 & 0.20 \\
\hline Duration of intervention & 0.003 & -0.005 to 0.011 & 0.403 & 0.00 \\
\hline Profession delivering intervention & -0.528 & -0.896 to -0.160 & $0.005^{* *}$ & 0.59 \\
\hline \multicolumn{5}{|l|}{ Multivariate analysis } \\
\hline Profession delivering intervention & -0.526 & -0.848 to -0.204 & $0.001^{* * *}$ & 1.00 \\
\hline Exercise intensity & -0.208 & -0.435 to 0.019 & 0.072 & \\
\hline \multicolumn{5}{|l|}{ Waist circumference } \\
\hline Mean age control group & -0.009 & -0.027 to 0.009 & 0.340 & 0.00 \\
\hline Mean age nutrition group & -0.010 & -0.027 to 0.008 & 0.448 & 0.00 \\
\hline Percentage men control & 0.003 & -0.014 to 0.020 & 0.742 & 0.00 \\
\hline Percentage men nutrition group & 0.006 & -0.005 to 0.018 & 0.291 & 0.00 \\
\hline Duration of intervention & 0.001 & -0.005 to 0.006 & 0.859 & 0.00 \\
\hline Profession delivering intervention & -0.369 & -0.759 to 0.021 & 0.064 & 0.00 \\
\hline \multicolumn{5}{|l|}{ Multivariate analysis } \\
\hline Profession delivering intervention & -0.365 & -0.762 to -0.033 & 0.072 & 0.00 \\
\hline Exercise intensity & 0.016 & -0.259 to 0.291 & 0.907 & \\
\hline
\end{tabular}

measured. ${ }^{28,31,32,38,44,46}$ Three studies used the Dietary Instrument for Nutrition Education (DINE) to assess fat (unsaturated and saturated) and fibre intake. ${ }^{28,31,32}$ One study providing hour-long weekly discussion groups did not find significant changes in anthropometric measures and also did not find significant changes in fat or fibre intake. ${ }^{28}$ Two studies delivered by keyworkers using a lifestyle manual, individually, which found small but significant improvements in anthropometric measures, found improvements in saturated fat and fibre intake, ${ }^{31}$ and improvements in saturated fat, fruit and vegetable intake. ${ }^{32}$ One study used the Block Fruit, Vegetable, and Dietary Fat Screeners to assess dietary change in an intervention providing healthy eating and weight-loss advice, delivered by research staff. ${ }^{38}$ This study did not find significant differences in dietary behaviours. A fifth study used a food frequency questionnaire, which appears validated in women in the general population; ${ }^{44}$ this questionnaire assessed the frequency of ten foods associated with better diet and ten foods associated with poorer diet. Individual psychoeducation and goal-setting significantly improved the score of foods associated with good diet compared with the control group, but not the score of foods associated with poorer diet. ${ }^{44}$ Finally, one trial involved using the Scottish Health Survey to assess whether providing free fruit and vegetables to families improved nutritional intake. ${ }^{46}$ Although improvements in fruit and vegetable intake were found, these were not sustained after the trial - and subsequent access to free fruit and vegetables - had ceased.

\section{Discussion}

To our knowledge this is the first systematic review to assess the impact of key components that comprise, and modes of delivery of, nutrition interventions on physical health measures of people with severe mental illness. We found that nutrition interventions improved anthropometric measures by reducing weight, BMI and waist circumference. Importantly, our review provides evidence about the most effective goals and delivery methods, including preventing weight gain from the initiation of antipsychotic therapy and the use of qualified health professionals such as dietitians to deliver individualised interventions. Our results indicated that nutrition interventions were most effective when delivered by a dietitian, with meta-regression analyses confirming this in multivariate models. These findings show a clear and important role for dietitians as part of the multidisciplinary mental health team.

Although the overall effect size for anthropometric measures was within the small to moderate range, it provided further evidence to support implementation of lifestyle interventions. Although between-group differences did not reach statistical significance, the larger effect size $(g=-0.61)$ seen in a pooled analysis of studies providing intervention in the early stages of antipsychotic therapy, where weight gain is most rapid, ${ }^{13}$ provides evidence for the achievability of goal 5 of the Healthy Active Lives declaration - that is, to restrict weight gain to no more than $7 \%$ of pre-illness weight in $75 \%$ of people experiencing first-episode psychosis, 2 years after commencing antipsychotic treatment. ${ }^{11}$ Targeting interventions to coincide with initiation of antipsychotic therapy is also warranted given the clinical benefits of preventing significant weight gain and metabolic deterioration. Given the large effect size $(g=-0.90)$ found for key anthropometric outcomes, nutrition interventions delivered by dietitians, in particular individualised counselling sessions, should have a central role in addressing cardiometabolic abnormalities and premature mortality in people with SMI. ${ }^{18,55}$ 
It was not possible to conduct a meta-analysis on nutritional intake owing to a lack of consensus on data collection methods together with the wide variety of outcome measures used. Nutritional intake can be notoriously difficult to assess accurately because of intake variability and the wide range of nutrients to consider, particularly if the assessment method is not targeted to the specific population. To date, there is no validated nutrition assessment tool developed for use in people living with severe mental illness, a significant gap in the literature requiring urgent attention. In addition, improvements in cardiometabolic health as a result of changes in nutritional intake, independent of weight change, have not been assessed in this population. This is a significant area requiring research, given the enduring weight challenges in this population.

Our results are broadly similar to previous non-pharmacological and physical activity intervention analyses completed by Álvarez-Jiménez et al, Bruins et al and Rosenbaum et al. ${ }^{12,56,57}$ Effect sizes (ES) from these analyses on anthropometric measures were respectively $Z=7.85$, ES $=0.63$ and standardised mean difference $=0.24$. However, ours is the first review to consider the impact of nutrition interventions and provides further recommendations for dietitian-led interventions incorporated from the early stages in SMI. As with the general population, nutrition interventions are most likely to be effective when combined with physical activity.

\section{Limitations and future research}

Several factors may have influenced the results obtained by this review, largely reflecting limitations in the primary studies. First, nutrition interventions were often delivered as part of a comprehensive lifestyle programme, thus participants were potentially receiving concomitant additional lifestyle interventions such as physical activity, which may have had a synergistic effect on participant outcomes. Although it was not possible to separate the impact of the nutrition intervention from additional components, we attempted to investigate the impact of nutrition interventions through a series of adjustments for potential confounders. For instance, for the primary outcome, our multivariate meta-regression analyses consistently demonstrated that nutrition interventions were most effective when delivered by a dietitian, even when we adjusted for concomitant exercise elements. Although the results from our subgroup analyses consistently demonstrated higher effect sizes of dietitian-led nutrition interventions and interventions delivered at antipsychotic initiation, these findings did not reach statistical significance after applying the Bonferroni correction. Although Bonferroni corrections are not routinely used in meta-analyses, ${ }^{58}$ we have opted for a conservative approach, and although our findings did not reach significance, they suggest more favourable outcomes and warrant further investigation. Second, the RCTs included a range of nutrition interventions including group education, individualised plans, practical shopping and cooking sessions and meal replacements. Subgroup analyses were run where possible, but were limited by the small numbers of studies and highly variable methods. Future research should seek to establish homogeneity in the use of appropriate outcome measures and nutrition interventions. Third, the potential for significant effects on relevant biochemical measures may have been affected by the short duration of interventions in many studies and the target outcomes frequently being limited to anthropometric measures. Future long-term studies encompassing specific dietary strategies to target dyslipidaemia are required, with adequate follow-up to see the impact of nutrition interventions in this population. ${ }^{59}$ Fourth, we did encounter some evidence of publication bias, but after recalculating the effect sizes using Duval \& Tweedie's trim and fill method our results were broadly similar. ${ }^{26}$ Fifth, we did encounter moderate heterogeneity in some of our analyses. Nonetheless, our multivariate metaregression analyses explained all $\left(R^{2}=1.0\right)$ of the observed heterogeneity for weight and BMI meta-analyses results. We were, however, unable to explain the heterogeneity in waist circumference; high levels of variability and inaccuracies in waist circumference measuring may be a factor. ${ }^{60}$ Finally, we were unable to access data from some of the identified studies, reducing the number of studies included and sample sizes included in some of the meta-analyses. In addition, some studies did not clearly identify the specific psychiatric diagnoses of participants, limiting the ability to draw further conclusions regarding the potential impact of diagnosis on the intervention outcomes obtained.

Nevertheless, allowing for these caveats, the results of this meta-analysis offer hope to clinical teams and patients alike that providing nutrition interventions as part of a wider multidisciplinary lifestyle intervention can be effective in preventing weight gain, particularly when delivered by a dietitian. University dietetic programmes will also need to evolve to increase the knowledge and understanding of SMI.

\section{Study implications}

Nutritional intake is fundamental to weight management and future physical health. This systematic review and meta-analysis found that nutrition interventions significantly improved weight, BMI, waist circumference and glucose levels in people with SMI. Further, nutrition interventions delivered by dietitians, and those aiming to prevent weight gain at antipsychotic initiation had the largest effect sizes. The evidence supports the early inclusion of nutrition intervention in mental health service delivery to people with SMI. Further RCTs are required to determine the most effective nutrition intervention to optimise weight and cardiometabolic health in people with SMI.

\section{Scott B. Teasdale, BNutrDiet, Keeping the Body in Mind Program, South Eastern Sydney Local Health District, Bondi Junction, and School of Psychiatry, University of New South Wales, Sydney; Philip B. Ward, PhD, School of Psychiatry, University o New South Wales, Sydney, Schizophrenia Research Unit, South Western Sydney Local Health District, and Ingham Institute for Applied Medical Research, Liverpool, New South Wales, Australia; Simon Rosenbaum, PhD, School of Medical Sciences, University of New South Wales, Sydney; Katherine Samaras, MBBS, PhD, Department of Endocrinology, St Vincent's Hospital, and Diabetes and Obesity Department of Endocrinology, St Vincent's Hospital, and Diabetes and Obesity
Program, Garvan Institute of Medical Research, Darlinghurst, New South Wales, Australia; Brendon Stubbs, PhD, MCSP, Physiotherapy Department, South London and Maudsley National Health Service Foundation Trust, London, and Health Service and Population Research Department, Institute of Psychiatry, Psychology and} Neuroscience, King's College London, London, UK

Correspondence: Scott Teasdale, Bondi Community Centre, 26 Llandaff St, Bondi Junction, NSW 2022, Australia. Email: Scott.Teasdale@sesiahs.health. nsw.gov.au

First received 20 Oct 2015; final revision 31 Aug 2016, accepted 6 Sep 2016

\section{References}

1 Dipasquale S, Pariante CM, Dazzan P, Aguglia E, McGuire P, Mondelli V. The dietary pattern of patients with schizophrenia: a systematic review. J Psychiatr Res 2013; 47: 197-207.

2 Jerome GJ, Young DR, Dalcin A, Charleston J, Anthony C, Hayes J, et al. Physical activity levels of persons with mental illness attending psychiatric rehabilitation programs. Schizophr Res 2009; 108: 252-7.

3 Cooper J, Mancuso SG, Borland R, Slade T, Galletly C, Castle D. Tobacco smoking among people living with a psychotic illness: the second Australian Survey of Psychosis. Aust NZ J Psychiatry 2012; 46: 851-63.

4 Bahorik AL, Newhill CE, Queen CC, Eack SM. Under-reporting of drug use among individuals with schizophrenia: prevalence and predictors. Psychol Med 2014; 44: 61-9. 
5 Treuer T, Hoffmann VP, Chen AK, Irimia V, Ocampo M, Wang G, et al. Factor associated with weight gain during olanzapine treatment in patients with schizophrenia or bipolar disorder: results from a six-month prospective, multinational, observational study. World J Biol Psychiatry 2009; 10: 729-40.

6 Blouin M, Tremblay A, Jalbert ME, Venables H, Bouchard RH, Roy MA, et al. Adiposity and eating behaviors in patients under second generation antipsychotics. Obesity 2008; 16: 1780-7.

7 Yum SY, Caracci G, Hwang MY. Schizophrenia and eating disorders. Psychiat Clin N Am 2009; 32: 809-19.

8 Bonfioli E, Berti L, Goss C, Muraro F, Burti L. Health promotion lifestyle interventions for weight management in psychosis: a systematic review and meta-analysis of randomised controlled trials. BMC Psychiatry 2012; 12: 78

9 Curtis J, Watkins A, Rosenbaum S, Teasdale S, Kalucy M, Samaras K, et al Evaluating an individualized lifestyle and lifeskills intervention to prevent antipsychotic-induced weight gain in first-episode psychosis. Early Interv Psychiatry 2016; 10: 267-76.

10 Teasdale S, Harris S, Rosenbaum S, Watkins A, Samaras K, Curtis J, et al. Individual dietetic consultations in first episode psychosis: a novel intervention to reduce cardiometabolic risk. Community Ment Health J 2014; 11: $211-4$

11 Shiers D, Curtis J. Cardiometabolic health in young people with psychosis. Lancet Psychiatry 2014; 1: 492-4.

12 Rosenbaum S, Tiedemann A, Sherrington C, Curtis J, Ward PB. Physical activity interventions for people with mental illness: a systematic review and meta-analysis. J Clin Psychiatry 2014; 75: 964-74.

13 Alvarez-Jimenez M, Gonzalez-Blanch C, Crespo-Facorro B, Hetrick S, Rodriguez-Sanchez JM, Perez-Iglesias R, et al. Antipsychotic-induced weight gain in chronic and first-episode psychotic disorders. CNS Drugs 2008; 22 : 547-62.

14 Correll CU, Manu P, Olshanskiy V, Napolitano B, Kane JM, Malhotra AK. Cardiometabolic risk of second-generation antipsychotic medications during first-time use in children and adolescents. JAMA 2009; 302: 1765-73.

15 Miller BJ, Paschall CB, Svendsen DP. Mortality and medical comorbidity among patients with serious mental illness. Psychiatr Serv 2006; 57: 1482-7.

16 Newcomer JW. Antipsychotic medications: metabolic and cardiovascular risk J Clin Psychiatry 2006; 68: 8-13.

17 Hennekens $\mathrm{CH}$, Hennekens AR, Hollar D, Casey DE. Schizophrenia and increased risks of cardiovascular disease. Am Heart J 2005; 150: 1115-21.

18 Vancampfort D, Stubbs B, Mitchell AJ, De Hert M, Wampers M, Ward PB, et al. Risk of metabolic syndrome and its components in people with schizophrenia and related psychotic disorders, bipolar disorder and major depressive disorder: a systematic review and meta-analysis. World Psychiatry 2015; 14 339-47.

19 Franz MJ, VanWormer JJ, Crain LL, Boucher JL, Histon T, Caplan W, et al. Weight-loss outcomes: a systematic review and meta-analysis of weight-loss clinical trials with a minimum 1-year follow-up. J Am Diet Assoc 2007; 107: 1755-67.

20 Ajala O, English P, Pinkney J. Systematic review and meta-analysis of different dietary approaches to the management of type 2 diabetes. Am J Clin Nutr 2013; 97: 505-16.

21 Yamaoka K, Tango T. Effects of lifestyle modification on metabolic syndrome: a systematic review and meta-analysis. BMC Med 2012; 10: 138.

22 Moher D, Liberati A, Tetzlaff J, Altman DG, PRISMA Group. Preferred reporting items for systematic reviews and meta-analyses: the PRISMA statement. Ann Intern Med 2009; 151: 264-9.

23 Critical Appraisal Skills Programme. CASP Randomised Controlled Trial Checklist. CASP, 2014

24 Begg CB, Mazumdar M. Operating characteristics of a rank correlation test for publication bias. Biometrics 1994; 50: 1088-101.

25 Egger M, Davey Smith G, Schneider M, Minder C. Bias in meta-analysis detected by a simple, graphical test. BMJ 1997; 315: 629-34.

26 Duval S, Tweedie R. Trim and fill: a simple funnel-plot-based method of testing and adjusting for publication bias in meta-analysis. Biometrics 2000; 56: $455-63$

27 Alvarez-Jimenez M, Gonzalez-Blanch C, Vazquez-Barquero JL, Perez-Iglesias R Martinez-Garcia $\mathrm{O}$, Perez-Pardal $\mathrm{T}$, et al. Attenuation of antipsychotic-induced weight gain with early behavioral intervention in drug-naive first-episode psychosis patients: a randomized controlled trial. J Clin Psychiatry 2006; 67 1253-60.

28 Attux C, Martini LC, Elkis H, Tamai S, Freirias A, Camargo MD, et al. A 6-month randomized controlled trial to test the efficacy of a lifestyle intervention for weight gain management in schizophrenia. BMC Psychiatry 2013; 13: 60 .
29 Brar JS, Ganguli R, Pandina G, Turkoz I, Berry S, Mahmoud R. Effects of behavioral therapy on weight loss in overweight and obese patients with schizophrenia or schizoaffective disorder. J Clin Psychiatry 2005; 66: 205-12.

30 Brown C, Goetz J, Hamera E. Weight loss intervention for people with serious mental illness: a randomized controlled trial of the RENEW program. Psychiatr Serv 2011; 62: 800-2.

31 Brown S, Chan K. A randomized controlled trial of a brief health promotion intervention in a population with serious mental illness. J Ment Health 2006 15: $543-9$.

32 Brown S, Smith E. Can a brief health promotion intervention delivered by mental health key workers improve clients' physical health: a randomized controlled trial. J Ment Health 2009; 18: 372-8.

33 Cordes J, Thunker J, Regenbrecht G, Zielasek J, Correll CU, Schmidt-Kraepelin $C$, et al. Can an early weight management program (WMP) prevent olanzapine (OLZ) induced disturbances in body weight, blood glucose and lipid metabolism? Twenty-four-and 48-week results from a 6-month randomized trial. World J Biol Psychiatry 2014; 15: 229-41.

34 Daumit GL, Dickerson FB, Wang NY, Dalcin A, Jerome GJ, Anderson CAM, et al. A behavioral weight-loss intervention in persons with serious mental illness. N Engl J Med 2013; 368: 1594-602.

35 Evans S, Newton R, Higgins S. Nutritional intervention to prevent weight gain in patients commenced on olanzapine: a randomized controlled trial. Aust NZ J Psychiatry 2005; 39: 479-86.

36 Forsberg KA, Bjorkman T, Sandman PO, Sandlund M. Influence of a lifestyle intervention among persons with a psychiatric disability: a cluster randomised controlled trial on symptoms, quality of life and sense of coherence. J Clin Nurs 2010; 19: 1519-28.

37 Gillhoff K, Gaab J, Emini L, Maroni C, Tholuck J, Greil L. Effects of a multimodal lifestyle intervention on body mass index in patients with bipolar disorder: a randomized controlled trial. Prim Care Companion J Clin Psychiatry 2010; 12

38 Goldberg RW, Reeves G, Tapscott S, Medoff D, Dickerson F, Goldberg AP, et al. 'MOVE!': Outcomes of a weight loss program modified for veterans with serious mental illness. Psychiatr Serv 2013; 64: 737-44.

39 Hjorth P, Davidsen AS, Kilian R, Pilgaard Eriksen S, Jensen SO, Sorensen HO et al. Improving the physical health of long-term psychiatric inpatients. Aust NZ J Psychiatry 2014; 48: 861-70.

40 Iglesias-Garcia C, Toimil-Iglesias A, Alonso-Villa MJ. Pilot study of the efficacy of an educational programme to reduce weight, on overweight and obese patients with chronic stable schizophrenia. J Psychiatr Ment Health Nurs 2010; 17: 849-51.

41 Jean-Baptiste M, Tek C, Liskov E, Chakunta UR, Nicholls S, Hassan AQ, et al. A pilot study of a weight management program with food provision in schizophrenia. Schizophr Res 2007; 96: 198-205.

42 Kwon JS, Choi JS, Bahk WM, Yoon Kim C, Hyung Kim C, Chul Shin Y, et al. Weight management program for treatment-emergent weight gain in olanzapine-treated patients with schizophrenia or schizoaffective disorder: a 12-week randomized controlled clinical trial. J Clin Psychiatry 2006; 67 $547-53$

43 Littrell KH, Hilligoss NM, Kirshner CD, Petty RG, Johnson CG. The effects of an educational intervention on antipsychotic-induced weight gain. J Nurs Scholarsh 2003: 35: 237-41.

44 Lovell K, Wearden A, Bradshaw T, Tomenson B, Pedley R, Davies LM, et al. An exploratory randomized controlled study of a healthy living intervention in early intervention services for psychosis: the INTERvention to encourage ACTivity, improve diet, and reduce weight gain (INTERACT) study. J Clin Psychiatry 2014; 75: 498-505.

45 Mauri M, Simoncini M, Castrogiovanni S, Iovieno N, Cecconi D, Dell'Agnello $\mathrm{G}$, et al. A psychoeducational program for weight loss in patients who have experienced weight gain during antipsychotic treatment with olanzapine. Pharmacopsychiatry 2008; 41: 17-23.

46 McCreadie RG, Kelly C, Connolly M, Williams S, Baxter G, Lean M, et al. Dietary improvement in people with schizophrenia: randomised controlled trial. Br J Psychiatry 2005; 187: 346-51.

47 McKibbin CL, Patterson TL, Norman G, Patrick K, Jin H, Roesch S, et al. A lifestyle intervention for older schizophrenia patients with diabetes mellitus: a randomized controlled trial. Schizophr Res 2006; 86: 36-44.

48 Milano W, Grillo F, Del Mastro A, De Rosa M, Sanseverino B, Petrella C, et al. Appropriate intervention strategies for weight gain induced by olanzapine: $a$ randomized controlled study. Adv Ther 2007; 24: 123-34.

49 Scocco $\mathrm{P}$, Longo $\mathrm{R}$, Caon $\mathrm{F}$. Weight change in treatment with olanzapine and a psychoeducational approach. Eat Behav 2006; 7: 115-24.

50 Usher K, Park T, Foster K, Buettner P. A randomized controlled tria undertaken to test a nurse-led weight management and exercise intervention designed for people with serious mental illness who take second generation antipsychotics. J Adv Nurs 2013; 69: 1539-48. 
51 Weber M, Wyne K. A cognitive/behavioral group intervention for weight loss in patients treated with atypical antipsychotics. Schizophr Res 2006 83: 95-101.

52 Wu MK, Wang CK, Bai YM, Huang CY, Lee SD. Outcomes of obese, clozapinetreated inpatients with schizophrenia placed on a six-month diet and physical activity program. Psychiatr Serv 2007. 58: 544-50.

53 Alvarez-Jimenez M, Martinez-Garcia O, Perez-Iglesias R, Ramirez ML, Vazquez-Barquero JL, Crespo-Facorro B. Prevention of antipsychotic-induced weight gain with early behavioural intervention in first-episode psychosis: 2-year results of a randomized controlled trial. Schizophr Res 2010; 116: $16-9$.

54 McKibbin CL, Golshan S, Griver K, Kitchen K, Wykes TL. A healthy lifestyle intervention for middle-aged and older schizophrenia patients with diabetes mellitus: a 6-month follow-up analysis. Schizophr Res 2010; 121: 203-6.

55 Walker ER, McGee RE, Druss BG. Mortality in mental disorders and global disease burden implications: a systematic review and meta-analysis. JAMA Psychiatry 2015; 72: 334-41.
56 Alvarez-Jimenez M, Hetrick SE, Gonzalez-Blanch C, Gleeson JF, McGorry PD. Non-pharmacological management of antipsychotic-induced weight gain: systematic review and meta-analysis of randomised controlled trials. Br J Psychiatry 2008; 193: 101-7.

57 Bruins J, Jorg F, Bruggeman R, Slooff C, Corpeleijn E, Pijnenborg M. The effects of lifestyle interventions on (long-term) weight management, cardiometabolic risk and depressive symptoms in people with psychotic disorders: a meta-analysis. PloS One 2014; 9: e112276.

58 Higgins JPT, Green S. Multiplicity in systematic reviews. In Cochrane Handbook for Systematic Reviews of Interventions:16.7.2. Cochrane Collaboration, 2011

59 Huang J, Frohlich J, Ignaszewski AP. The impact of dietary changes and dietary supplements on lipid profile. Can J Cardiol 2011; 27: 488-505.

60 Panoulas VF, Ahmad N, Fazal AA, Kassamall RH, Nightingale P, Gitas GD, et al. The inter-operator variability in measuring waist circumference and its potential impact on the diagnosis of the metabolic syndrome. Postgrad Med J 2008; 84: 344-7.

\section{psychiatry in history}

James Foulis Duncan (1812-1895)

\author{
Brendan D. Kelly
}

James Foulis Duncan was the last of the Duncan family to own Farnham House, a private asylum in Finglas, County Dublin, Ireland. He served as president of the King and Queen's College of Physicians in Ireland (1873-1875) and of the Medico-Psychological Association (MPA) in 1875.

Duncan's mother died when he was young so he grew up with his father at the asylum. They ate with the patients and Duncan's earliest lessons in Latin, mathematics and science were provided by patients whom he later described as the best and noblest persons of our race, of gifted intellect and high attainment.

Building on this unusual but effective foundation, Duncan graduated from Trinity College Dublin in 1837. Throughout his medical and psychiatric career, Duncan was acutely socially aware, especially of the effects of poverty on health. He supported the use of scientific comparisons to test treatments (in language that prefigured later ideas about clinical trials) and was highly religious in outlook, as evidenced in his 1852 publication, God in Disease, or, The Manifestations of Design in Morbid Phenomena.

In his presidential address to the MPA in 1875, Duncan vehemently denounced a great many features of 19th-century life, ranging from the substitution of machinery for handicraft labour to the employment of children in factories and consequent loosening of family bonds. He was not, however, a man to be easily defeated, not even by great, unstoppable forces of history. Duncan saw education as the answer to all of these problems and advocated for better medical education, broader public education, and moral education of the young, which he felt held the greatest hope for preventing mental disorder.

Duncan typified a certain model of 19th-century asylum doctor: enterprising, powerful, prolific and keen to promote asylum medicine in the eyes of other doctors and the public. He died on 2 April 1895 at the age of 83 years. Obituaries in the British Medical Journal and Medical Press noted the professional esteem in which he was held, as well as his devotion to the promotion of religion and his reputation as a man of charity.

Image reproduced by kind permission of the Royal college of Physicians of Ireland. 